\title{
DBET: Demand based Energy efficient Topology using P-MANET
}

\author{
M.Thamizarivu and S.Vijayakumar \\ Dept. of Electronics and Communication Engineering \\ Ganadipathy Tulsi's Jain Engineering College \\ Vellore, Tamilnadu, India - 632102.
}

\begin{abstract}
Energy efficient topology in Ad-hoc networks be achieved mainly in two different ways. In the first method, network maintains a small number of nodes to form a connected backbone and the remaining nodes sleep to conserve energy. This method is effective for low traffic networks. Energy efficiency in the second method is achieved by power control technique. This technique is effective in high traffic conditions. The first method is not effective in high traffic conditions. Similarly, the second method is not effective in low traffic networks. So, Existing author's introduce a Demand Based Energy efficient Topology (DBET) to reduce the energy consumption for mobile ad hoc network, by dynamically adjusting the topology for various network traffic conditions. In this paper, Author propose efficient power saving mac protocol (P-MANET) based on a Multi-hop Time Synchronization Protocol to reduce the power consumption, transmission latency.
\end{abstract}

\section{General Terms}

Mobile Ad-hoc Network.

\section{Keywords}

Efficient power saving protocol, manet, ns2 simulator.

\section{INTRODUCTION}

Mobile Ad-hoc Networks (MANETs) are self-organizing, self-configuring and infrastructure-less multi-hop wireless networks, where each node communicates with other nodes directly or indirectly through intermediate nodes without any infrastructure. Such temporary networks can be used in battlefields, disaster areas, military applications, mining operations and robot data acquisition. Besides these characteristics they present challenges like limited energy, dynamic topology, low bandwidth and security. The description of the arrangement of the MANETs, called topology, is usually temporary or dynamically changed with time.

Energy consumption can be varied into different traffic conditions. Network keep small number of nodes and form a connected backbone, remaining nodes can be go to sleep state and conserve the energy. This can be used in low traffic conditions only. The power control technique can be used in high traffic conditions. To tolerate the various network traffic conditions, The demand based energy efficient topology can be introduced. In this paper, Author propose efficient power saving mac protocol based on multi-hop time synchronization protocol.

Power saving method is not effective in high traffic conditions. Similarly, the sleep mode method is not effective low traffic networks. as same time topology of the existing system is totally changed based on the traffic condition so large and low traffic have different parameters, so it is complicated to maintain the single topology. It has less reliable operation and more energy dissipation. Due to different topology router drops are very high. But Author propose P-MANET to reduce the power consumption for various network traffic conditions and also reduce the router overhead and bandwidth utilization. It increases the throughput and reduces broadcasting between nodes.

The rest of the paper is organized as follows: the next section provides a brief review of related work. The third section gives the design details of proposed work. Integration issues of DBET using P-MANET with routing protocol is discussed in the forth section. Simulation results along with discussion are provided in the section 5. The last and final section concludes the paper with same pointers to future research direction.

\section{RELATED WORK}

They briefly describe various techniques related to our work. Different techniques are used to reduce the power consumption. Now discuss about various methods to reduce the power consumption.

Madhavi W. Subbarao [2] develop an initial dynamic powerconscious routing scheme (MPR) to route a packet on a path that will require the least amount of total power. The main idea of MPR is to select the path between a given source and destination. As an initial approach, the distributed BellmanFord algorithm can be used to perform shortest" path routing with the cost functions as the link distances .Dynamic power consumption based on the alternating of 0 's and 1 's transmitting bits.

Ram and Regina Hain [3] consider the problem of adjusting the transmit powers of nodes in a multihop wireless network. They present two distributed heuristics that adaptively adjust node transmit powers namely Local Information No Topology (LINT) and Local Information Link State Topology (LILT). LINT uses locally available neighbor information collected by a routing protocol. Power adjustment based on the threshold value. LILT also uses the freely available neighbor information. In LILT, They introduce two protocol such as neighbor addition protocol, neighbor reduction protocol.

Power adjustment based on the adding the nodes, reducing the nodes. Gupta and Rao [1] introduce demand based energy efficient topology to reduce energy consumption for various 
network traffic conditions. The DBET can be divided into four phases.

Phase I: Independent set formation.

Phase II: Connecting the Independent Set

Phase III: Coordinator Withdraw

Phase IV: Local route customization with Power control technique

In phase-I, the node can be selected based on three factors such as stability factor, utility factor, energy factor. The selected node can be used for data transmission. In phase-II, the selected node can be form a connected backbone, remaining node can go to the sleep state and conserve the energy.

In phase-III, If first phase active nodes may move into a region that already has another first phase active node so that the region now has more than one first phase active nodes. These active nodes recognize this situation and one of them withdraws. In phase-IV, energy consumption can be reduced by choosing a lower energy cost path. The DBET uses only 1hop neighbor information.

\section{PROPOSED WORK}

The power consumption can be reduced by introducing the Efficient Power Saving MAC Protocol based on multi-hop time synchronization. It involves hibernation mechanism, beacon inhibition mechanism, low latency next hop selection mechanism. Let us discuss about three mechanism.

\subsection{Hibernation mechanism}

A node can waste the energy due to idle listening, collision, overhearing and control mechanism. This mechanism can eliminate the power consumption. In this mechanism, every node can be communicated with other node if the node can be in listen mode. Otherwise it can be in sleep mode. Every node receives $\mathrm{N}$ beacon intervals and form a cycle. Each node can enter the listen mode only once per cycle. The Hibernation mechanism can determine about the node can enter the listen mode in which beacon interval. This can be done by hash function such as SHA-1. Node utilizes the global hash function to transmit the MTIM frame and data packet in the listen mode. The MTIM frame can be used to inform for sending the data frame to corresponding node. This frame can be received in MTIM window. The data packet can be received in the data window.

In this mechanism, Collision, overhearing can be avoided by using the sequence of four operations RTS/CTS/DATA/ACK. The MTIM frame and Data frame can be sent into these sequence orders. The Network Allocation Vector can avoid the overhearing and save the power. For example, Nodes A, B want to send the data to Node $\mathrm{C}$ in Beacon interval 2. Node A and $\mathrm{C}$ can be communicate with each other in slots 1-4. At the same time, Node B wants to communicate to Node C. So Node B can send the packet in slots 5-8 to Node C. In this way, The collision and overhearing can be avoided. In NAV, If the channel is busy, the mobile node can stop sensing the channel and go to sleep state.

\subsection{Beacon inhibition mechanism}

The beacon inhibition mechanism can be used to reduce the number of collisions and increase the transmission efficiency of packets during MTIM window. A node can emit a more number of beacon message based on the threshold value. The
Threshold value can be estimated as the ratio of length of beacon window and time taken to send the beacon.

$$
\text { Threshold }(\mathrm{N})=\text { Length of beacon window }(\mathrm{L})
$$$$
\text { Time taken to send a beacon(t) }
$$

The mobile node can stop sensing for beacons if the total number of beacon messages less than the threshold value. For example, the BW is $4 \mathrm{~ms}$, and sending a beacon takes $0.5 \mathrm{~ms}$, then the threshold is 8.In this way, the power consumption can be reduced.

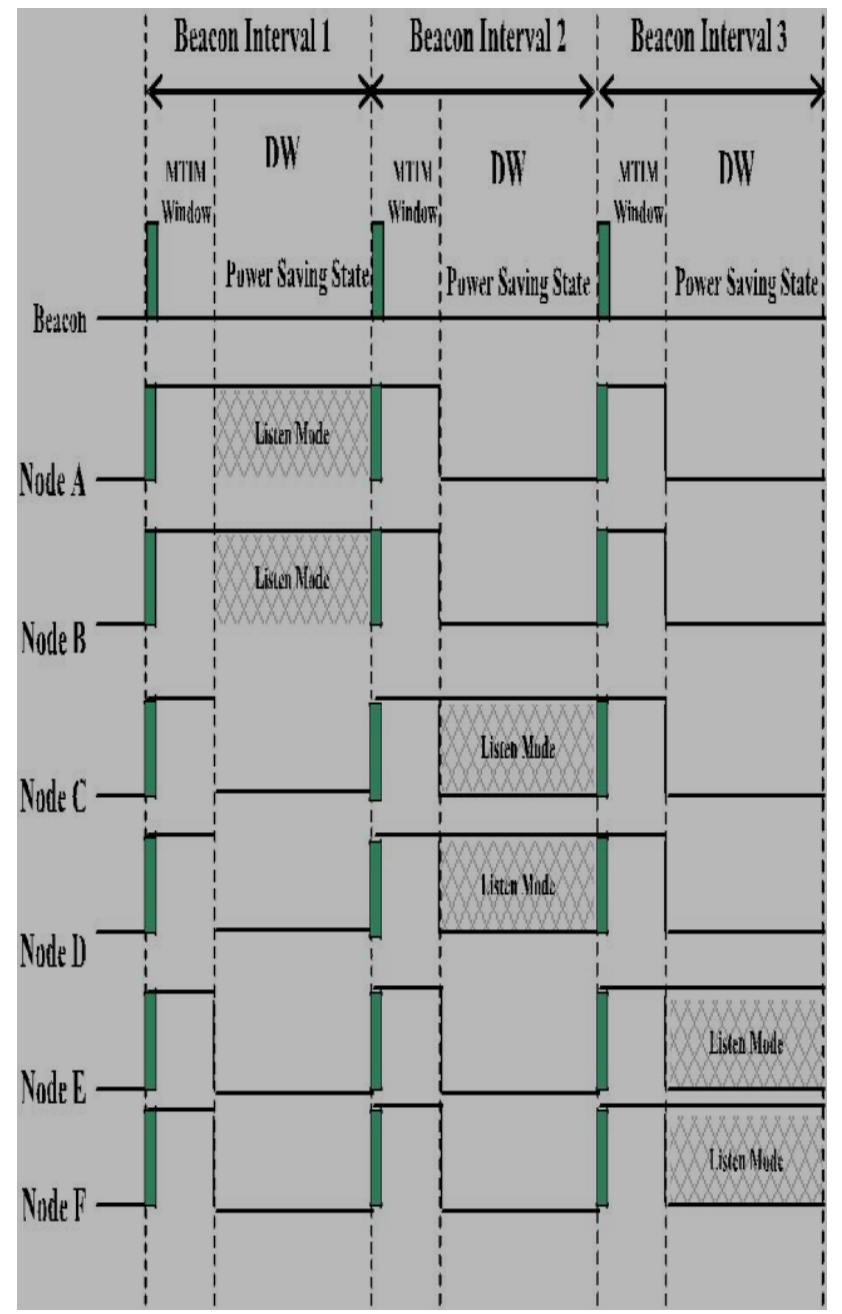

Fig 1. A structure of beacon interval of P-MANET with three intervals.

\subsection{Low latency next hop selection mechanism}

The low latency next hop selection mechanism can be used to find the path and reduce the power consumption. In this mechanism, on demand protocols such as AODV have been used. The neighbor's information can be maintained in the routing table. The mobile node can broadcast the route request RREQ packet to other nodes. 


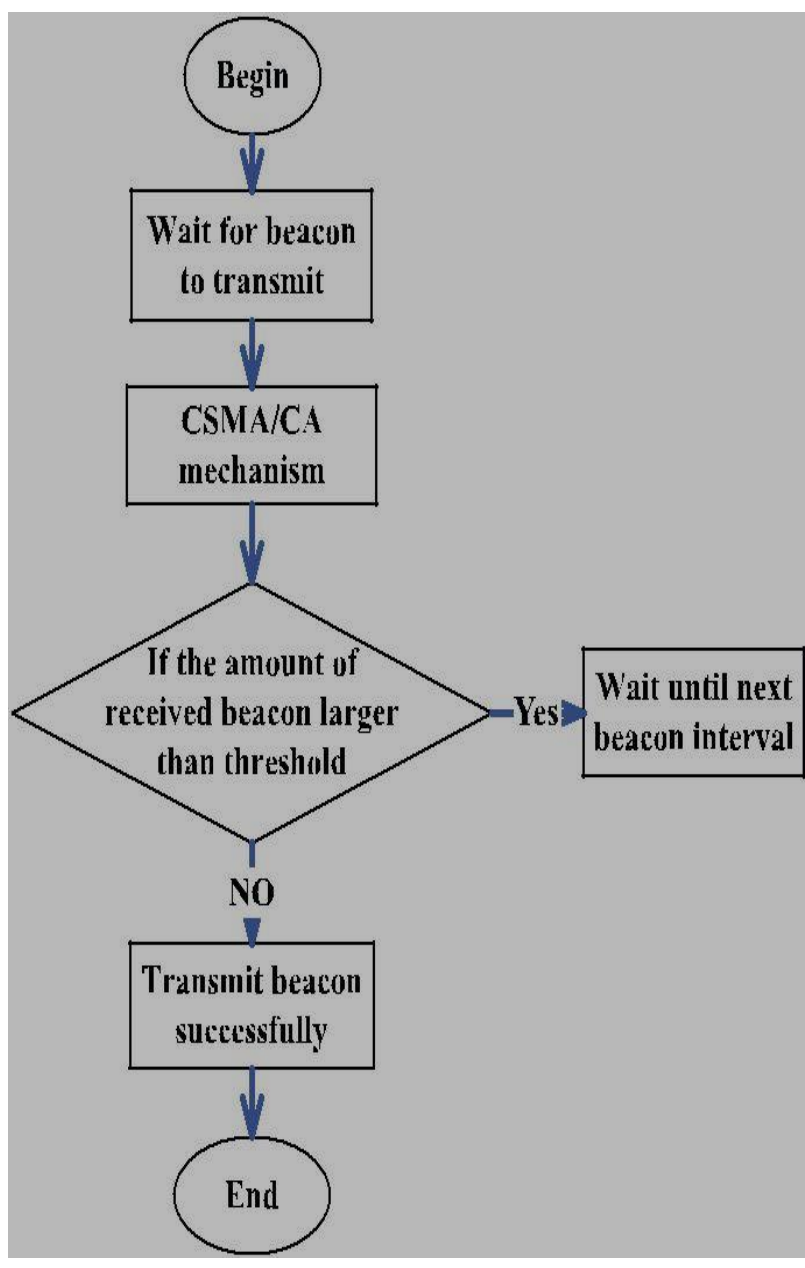

Figure 2. Flow chart of Beacon inhibition mechanism

The neighbor's can send the route reply RREP packet to mobile nodes. In this way, the route can be established. After establishing the route, packet can be sent. The route path is chosen as shortest path. The shortest path can be reduced the latency. The route discovery process fails if the source does not receive a RREP packet within maximum tolerable round trip time (MTRTT). MTRTT can be estimated on the basis of real-time measurements using exponential weighted moving average method. As the node is away or power off anytime, route maintenance and dissemination of energy information of the nodes is performed periodically by flooding some number of explore packets.

\section{INTEGRATION ISSUES}

For example, the mobile nodes can broadcast the beacon messages to other nodes in the beacon window because to know about whether it is in listen mode or not. Node A, B can be in listen mode at the beacon interval 1 . Node $\mathrm{C}$ can enter in listen mode at the beacon interval 2. Node A send the RREQ packet to node B. Node B forward the packet to node $\mathrm{C}$ if path is not found. Node C send RREP packet to node B. Node B forward the packet to node A. Path can be established.

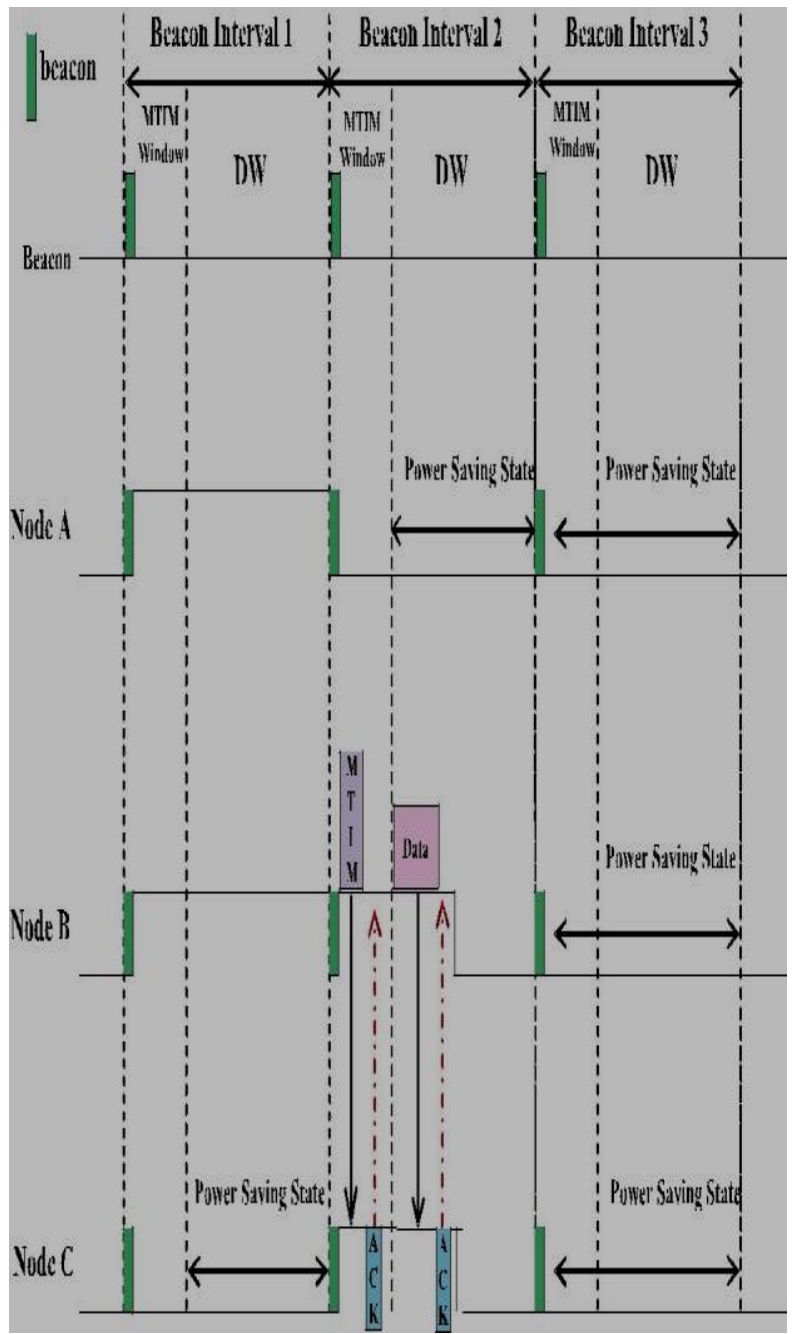

Fig 3. An example of transmission of packets

So Node A send the MTIM frame to node C in the MTIM window at the beacon interval 1 because to inform for sending the data frames. It receive the MTIM frame(RTS) at the beacon interval 2. After Node C send CTS packet to Node B. Again Node A sends Data frame to node $\mathrm{C}$ in the data window. Node $C$ receives the packet and sends the ACK to node A. In this way, communication can be done. Other nodes can communicate with the node A, the NAV schedule the time slots to communicate. Overhearing, collision can be avoided by using the similar of CAMA/CA mechanism. If the packet can be drop within the maximum tolerable round trip time, node send the explore packet to source node.

\section{SIMULATION RESULT}

The figure 4 gives the comparison between the existing work and the proposed work. Existing work is DBET. Proposed work is P-MANET. The existing work denoted as in the green line. Proposed work denoted as a red line. This is the graph result between mobility and delivery ratio. When mobility increases, delivery ratio has increases in P-MANET compared to DBET.

The figure 5. is the comparison of no of nodes and delay. When no of nodes increases, delay can be decreases in PMANET compared to DBET. The red line indicate existing 
work that is DBET. Green line indicate proposed work .

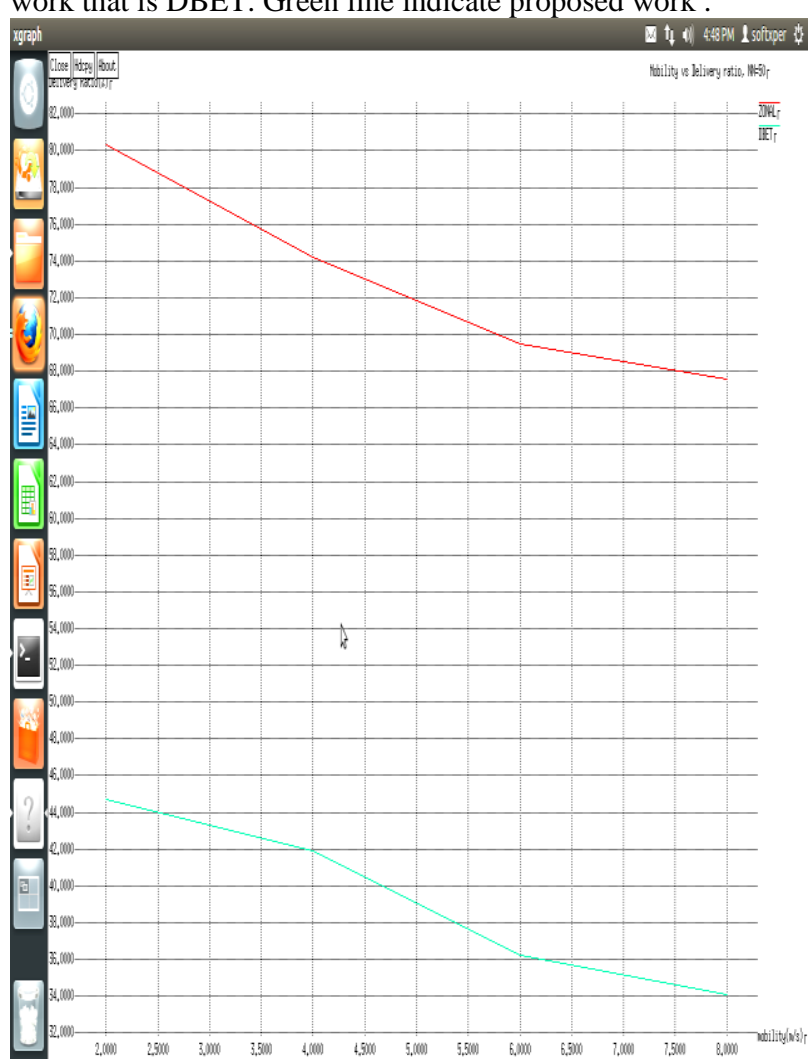

Figure 4. Mobility Vs Delivery ratio

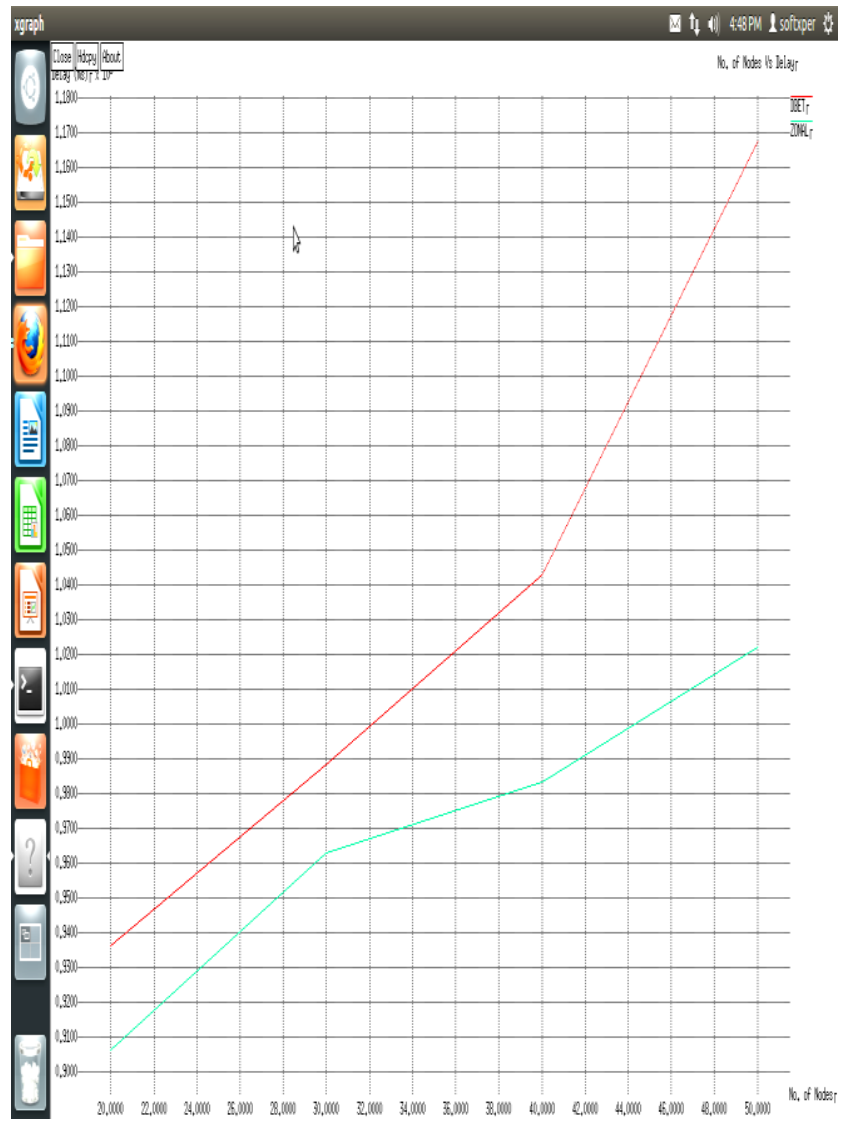

Fig.5. No of nodes Vs Delay

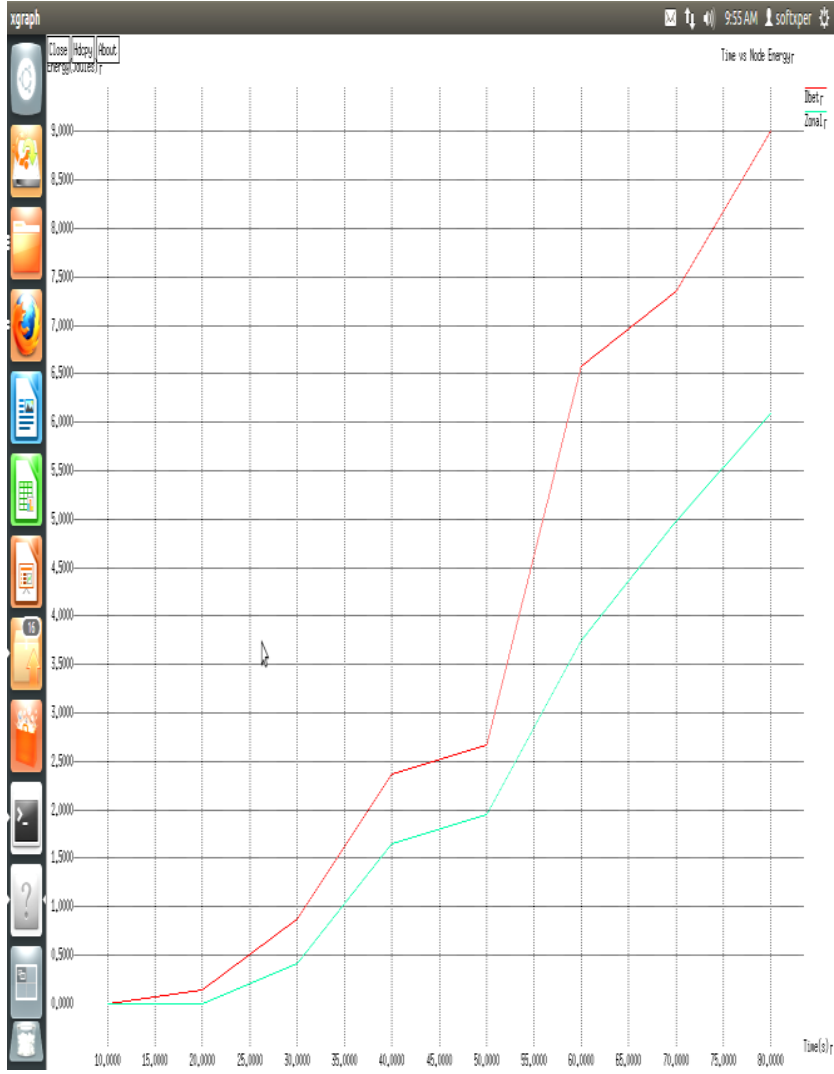

Fig.6. Time Vs Node Energy

The figure 6. is the comparison of time and node energy.The red line indicate as exixting work that is DBET.The Green line indicate proposed work that is P-MANET.When time taken to send a packet is too long ,node energy can be decreases in P-MANET compared to DBET.

\section{CONCLUSIONS}

The efficient power saving protocol based on multi-hop synchronization which involves hibernation mechanism, beacon inhibition mechanism, low latency next hop selection mechanism can be used to reduce the delay, more power consumption, overheating. Hibernation mechanism can be used to find that the node can enter into listen mode in which beacon interval and avoid collision, overhearing. Beacon inhibition mechanism can be used to solve beacon storm problem. Low latency next hop mechanism can be used to reduce the delay and find the shortest path. These mechanism can gives the $70 \%$ simulation result in the power saving, a low neighbor discovery time, and a low transmission latency with p-MANET for this project.

\section{FUTURE WORK}

Several issue related to P-MANET require further investigation. The authors are developing upper-layer protocols, such as a power-aware routing protocol and poweraware application protocols on the basis of the cross layer design. Therefore, the overall routing performance requires further evaluation. Power consumption and message overhead will be taken into account, with the expectation of obtaining a more scalable solution. 


\section{ACKNOWLEDGMENTS}

Author's would like to thank all for this hard-work and efforts in creating simulation environment in MANET, executing the simulations and producing the plots.

\section{REFERENCES}

[1] Hari Prabhat Gupta, and Rao, S. V. 2011. Demand Based Energy Efficient Topology for MANET's.

[2] Madhavi, W. Subbarao, 1999. Dynamic power-conscious routing for manets:An initial approach. In in IEEE Vehicular Technology Conference, pages 1232-1237.

[3] Ram Ramanathan, and Regina Hain, 2000. Topology control of multihop wireless networks using transmit power adjustment. In INFOCOM, pages 404-413.

[4] Stemm, M. Gauthier, P. Harada, D. Katz R. H. 1997. Reducing power consumption of network interfaces in hand-held devices. IEICE Transactions on Communications E80-B(8):1125-1131.

[5] Cano, J. C. Manzoni, P. 2001. Evaluating the energyconsumption reduction in a MANET by dynamically switching-off networkinterfaces. 6th IEEE Symposium on Computers and Communications (ISCC), Hammamet, Tunisia, 186-191.

[6] Zheng, R. Kravets, R. 2003. On-demand power management for ad hoc networks. IEEE Conference on Computer Communications (INFOCOM), San Francisco, USA,481-491.

[7] Chen, B. Jamieson, K. Balakrishnan, H. Morris, R 2003. Span: an energy efficient coordination algorithm for topology maintenance in ad hoc wireless networks. ACM/Springer Wireless Networks, San Francisco, USA,481-491.

[8] Zhang, T. Gurung, P. 2006. Van Den Berg E, Madhani S, Muttreja A. Silent networking for energy constrained nodes. Computer Communications, 29(17):3445-3454.

[9] Belghith, A. Akkari, W. Bonnin, J. M. 2007. Traffic aware power saving protocol in multi-hop mobile ad-hoc networks. Journal of Networks, 2(4):1-13.

[10] Belghith, A. Akkari, W. 2008. Neighborhood aware power saving mechanisms for ad hoc networks. IEEE Conference on Local Computer Networks (LCN), 114121

[11] Xu, Y. Heidemann, J. Estrin, D. 2000. Adaptive energyconserving routing for multihop ad hoc networks. Tech. Rep. 527, USC/Information Sciences Institute.
[12] Tseng, Y. C. Hsu, C. S. 2003. Hsieh T-Y. Power-saving protocols for IEEE 802.11-based multi-hop ad hoc networks. Computer Networks,43(3):317-337.

[13] Jiang, J. R. Tseng, Y. C. Hsu, C. S. Lai, T. H. 2005. Quorum-based asynchronous power-saving protocols for IEEE 802.11 adhoc networks. Mobilen Networks and Applications, 10(1-2):169-181.

[14] Zheng, R. Hou, J. C. Sha, L. 2006. Optimal block design for asynchronous wake-up schedules and its applications in multihop wireless networks. IEEE Transactions on Mobile Computing, 5(9):1228-1241

[15] Wu, S. H. Chen, M. S. Chen, C. M. 2008. Fully adaptive power saving protocols for ad hoc networks using the hyper quorum system. IEEE International Conference on Distributed Computing Systems (ICDCS), 785-792.

[16] Chao, M. Sheu, J. P. Chou, I. C. 2006. An adoptive quorum-based energy conserving protocol for IEEE 802.11 ad hoc networks. IEEE Transactions On Mobile Computing, 5(5):560-570.

[17] Huang, L. Lai, T. H. 2002. On the scalability of IEEE 802.11 ad hoc networks. ACM Mobile Ad Hoc Networking and Computing (MOBIHOC), 173-182.

[18] Lai, T. H. Zhou, D. 2003 Efficient and scalable IEEE 802.11 ad hoc mode timing synchronization function. IEEE Conference Advanced Information Networking and Applications (AINA),318-323

[19] Perkins, C. E. Bhagwat, P. 1994. Highly dynamic destination-sequenced distance-vector routing (DSDV) for mobile computers. ACM SIG on Data Communications (SIGCOMM) ,24(4):234-244.

[20] Sheu, J. P. Chao, C. M. Sun, C. W. 2004. A clock synchronization algorithm for multi-hop wireless ad hoc networks. IEEE International Conference on Distributed Computing Systems (ICDCS), 574-581.

[21] Chen, G. N. Wang, C. Y. Hwang, R. H. 2007. MTSP: Multi-hop Time Synchronization Protocol for IEEE 802.11 wireless adhoc network. Journal of Information Science and Engineering, 23(4):969 983.

[22] Rauschert, P. Honarbacht, A. and Kummert, A. 2004. On the IEEE 802.11 IBSS and its timer synchronization function in multi-hop ad hoc networks. IEEE International Symposium on Wireless Communication Systems, 304-308. 